\title{
Safety and Effectiveness of Low-Level Laser Therapy in Patients With Knee Osteoarthritis: A Systematic Review and Meta-analysis
}

\author{
Seyed Mansour Rayegani ${ }^{1}$, Seyed Ahmad Raeissadat ${ }^{1}$, Saeed Heidari², Mohammad Moradi-Joo ${ }^{3,4^{*}}$ \\ ${ }^{1}$ Physical Medicine and Rehabilitation Research Center, Shahid Beheshti University of Medical Sciences, Tehran, Iran \\ ${ }^{2}$ Health Technology Assessment, Social Development \& Health Promotion Research Center, Kermanshah University of \\ Medical Sciences, Kermanshah, Iran \\ ${ }^{3}$ Cancer Research Center, Shahid Beheshti University of Medical Sciences, Tehran, Iran \\ ${ }^{4}$ Iran Health Insurance Organization, Tehran, Iran
}

\section{*Correspondence to \\ Mohammad Moradi-Joo. MS.c; \\ Health Technology Assessment, Iran \\ Health Insurance Organization, \\ Tehran, Iran. \\ Tel: +98 9177192294; \\ Fax : +98 2122748001 \\ Email: moradijoo@gmail.com}

Published online 29 August 2017

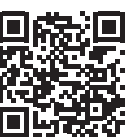

\begin{abstract}
Introduction: Low-level laser therapy (LLLT) was introduced as an alternative non-invasive treatment for osteoarthritis, but its effectiveness is still controversial. The main objective of this article was to determine the safety and efficacy of LLLT in patients with knee osteoarthritis (KOA). Methods: In order to gather evidence, main medical databases as well as relevant websites were browsed without time limit. We searched with appropriate keywords and strategies. After quality assessment of studies, study data were extracted by two reviewers. Standard mean difference proposed through inverse variance was used in the meta-analysis using the random-effects model. Twelve values were used for the evaluation of heterogeneity.

Results: A total of 823 studies, 14 randomized controlled trials (RCTs) were selected after final review. There was a significant difference between LLLT and placebo in pain at rest $(P=0.02)$, pain at activity $(P=0.01)$, total pain $(P=0.03)$, WOMAC function $(P=0.01)$, WOMAC stiffness $(P=0.02)$ and WOMAC total $(P<0.0001)$ in favor of the LLLT. There was no significant difference between LLLT and Placebo in WOMAC pain $(P=0.09)$ and range of motion $(P=0.1)$.

Conclusion: In spite of some positive findings, this meta-analysis lacked data on how LLLT effectiveness is affected with important factors: wavelength, energy density, treatment duration, numbers of sessions the treatment, severity of KOA and site of application.

Keywords: Knee, Osteoarthritis; Low level laser therapy; Effectiveness; Meta-analysis.
\end{abstract}

\section{Introduction}

Osteoarthritis is one of the most common diseases which has affected many people; this disease increases with age. In general, it may be said that approximately $14 \%$ of adults in United States and $40 \%$ of the population under 65 years old in Britain and Australia suffer from this disease; this disease prevalence is growing. ${ }^{1}$ Osteoarthritis is an example of physical and motor disability and one of the most important causes of functional disabilities in individuals, and imposes significant costs to individuals and consequently, to society. ${ }^{2}$ Knee osteoarthritis (KOA) is one of the most common osteoarthritis types and is associated with symptoms such as knee stiffness, pain, and limited movement range of knee joint. These conditions lead to physical function impairment, limited independence in doing daily life activities, decreased quality of life, and other disabilities. ${ }^{3,4}$

KOA is also known as arthritis or knee joint stiffness; it mainly affects the articular cartilage. The articular cartilage is a soft tissue which is inside the joint and between bones; in the case of its damage, the bones will rub on each other and extended bone will be created around the joint. Ultimately, the ligaments and muscles around the joint will be narrowed and weakened. ${ }^{1-4}$ There are several different treatments to control this disease (pharmacotherapy, surgery, physiotherapy, exercise therapy). It seems that the use of these methods depends on disease stage and patient's specific condition. In general, however, the logic and philosophy of these methods is relieving patient from pain, make him/her independent in doing daily life activities, and improving his/her life. ${ }^{5}$ Due to numerous complications which are associated with using non-steroidal drugs, using them to control this disease is limited. ${ }^{5}$ Recently, other therapies which are based on rehabilitation methods (such as exercise therapy, tensile muscle movement, electrical stimulation, acupuncture, and heat therapy) are more considered by physicians than using non-steroidal drugs. ${ }^{6}$ Low-level laser therapy (LLLT) is one of the safe and non-invasive methods which has recently attracted the attention of

Please cite this article as follows: Rayegani SM, Raeissadat SA, Heidari S, Moradi-Joo M. Safety and effectiveness of low-level laser therapy in patients with knee osteoarthritis: a systematic review and meta-analysis. / Lasers Med Sci. 2017;8(Suppl 1):S12-S19. doi:10.15171/ jlms.2017.s3. 
many researchers and specialists for treatment of KOA. ${ }^{7}$ Low-power laser therapy is a therapeutic approach which uses low intensity light-emitting in range of 540$830 \mathrm{~nm}$ light. It seems that the therapeutic effects of this procedure emerge by photochemical reactions which change permeability of cell membrane, increase buildup of mRNA, and lead to cell proliferation. The causes of these effects are not heat; like in surgery laser. The LLLT is used in many different diseases and mainly in pain control. Simply, it may be said that there are two types of lasers: low power and high power. The high-power lasers cut the tissue and release heat. However, the lowpower lasers do not release heat and do not damage the tissue; they have the potential to produce photochemical reactions and improve the metabolism of cells. They are named low power lasers because they have a density of less than $5.0 \mathrm{~W} / \mathrm{cm}^{2}$; they are also referred to as cold lasers or soft lasers. These lasers react in tissue and without causing heat, stimulate or inhibit the cells. ${ }^{8}$

After radiation of laser photons into cells, the cellular response begins with the activation of photoacceptors which are in the respiratory chain in mitochondria. Then, the cell redox and cell membrane state change, calcium displaces, $\mathrm{pH}$ changes, CAMP is activated, and DNA is dubbed; all of these lead to protein synthesis. In this way, the cellular responses extend from cell surface to tissue and organ surfaces and cause effects such as anti-inflammation, anti-edema and swelling, analgesia, cell proliferation, neovascularization and accelerated recovery, shifting metabolism to aerobic, and balancing immune system. In general, the physiological responses of tissue to low-power lasers include biological stimulation of system, effects on immune system, anti-inflammatory and anti-edema effects, effects on vessels and circulation, effects on lymph, effect on wound healing, analgesic effect, and effects on nerves. ${ }^{8}$ However, it seems that there are some uncertainties regarding the use of this therapeutic approach in people with KOA and the results of some studies are contradictory. ${ }^{9}$

Despite the broad clinical application of LLLT and also due to numerous randomized controlled trial (RCT) papers in terms of safety and efficacy of low power laser in people with $\mathrm{KOA}$, the results of these articles are sometimes in contradiction with each other and its effectiveness is still in doubt. On the other hand, there are few systematic and meta-analyses studies that clarify the efficacy and safety of LLLT in these patients. The last systematic study that has been conducted in this area in recent years belongs to Huang et al. ${ }^{10}$ However, the study does not encompass some related articles, does not consider follow-up period of outcomes in the analysis and does not analyze consequences such as pain at rest, pain during activities and quality of life. Accordingly, we aimed to alleviate some of these limitations, to have a more comprehensive analysis of the safety and effectiveness of low power laser in people with KOA and ultimately contribute to an evidence-based decision making and policy on the use of this technology in this group of patients.

\section{Methods}

This study conforms to all PRISMA guidelines and reports the required information accordingly. We performed a systematic review of best evidence using Cochrane guidelines. Our structured question for this review was as follows in Box 1.

Search for Evidence and Article Selection

We searched the most important and appropriate electronic medical databases including MEDLINE, PubMed, Cochrane library, Science Direct, Trip, Google Scholar, Thomson Reuters (formerly ISI) Web of Knowledge, SCOPUS and EMBASE as well as relevant websites were browsed without time constraint up to September 2016. The Me SH system was used, as well as 'AND' and 'OR' between words of the same meaning and concept i.e. Low Level, Low Power, Laser Therapy, LLLT, LPLL, osteoarthritis and knee. The collected papers were organized in Endnote software. After deleting duplicate articles, 2 reviewers independently assessed the titles and abstracts of the search results and selected potentially relevant studies according to our main question (Box 1). The articles that were deemed to be irrelevant to the research objectives were excluded. After collecting the full texts of articles which were related to the objectives of this study, the references of these articles were reviewed. The related references were identified and their full texts were reviewed. All stages of obtaining evidence and selecting studies were independently conducted by 2 authors. The disputes between them were resolved by discussion and by third party, if needed.

\section{Inclusion and Exclusion Criteria}

The inclusion criteria included (1) clinical trials articles, (2) people with KOA who were identified and approved by American College of Rheumatology indices, (3) the articles which compared low-power and LLLT with placebo laser, (4) articles which examined consequences such as pain, stiffness, performance, activity, and quality of life, and (5) articles which were published in English or Persian. The exclusion criteria included (1) fundamental research or studies on animals, (2) review articles, (3) articles which had not related statistical and clinical data, (4) articles which used LLLT or placebo laser along with another intervention, (5) duplicate articles which had up-to-date versions. Since the full text of all articles was available and we managed to get all of full articles, none of

\section{Box 1. Components of Structured Question}

- $\quad$ Population: People with KOA;

- Intervention: Low-level(power) laser therapy;

- Comparator: Placebo Laser Therapy;

- $\quad$ Outcome: Pain relief, activity, range of motion, adverse side effects;

- $\quad$ Type of studies: RCTs and observational studies 
them was excluded.

Quality Assessment

The Cochrane's Paper Quality Assessment Checklist was used to evaluate the quality of articles; this process was carried out independently by 2 authors and the disputes between them were resolved by discussion and by third party, if needed. Since this tool evaluates 7 aspects of bias risk, therefore, a 10-point scale was considered for article quality: scores above 7 indicate high quality, scores between 5 and 7 indicate medium quality of paper, and scores less than 5 indicate low quality of article. In dimensions 1 to 6 , in general, the articles with low bias risk scored 1.5, the obscure bias risk scored 0.75 , and the articles with high bias risk scored 0 . In seventh dimension, the low bias risk scored 1 , the obscure bias risk scored 0.5 , and the high bias risk scored 0 .

\section{Data Collection}

A special form was created in Excel 2013 to extract data from final articles. The needed data including general information (including title of article, year of publication, country, corresponding author) and specific information (including population size, sample size, type of study, statistical data related to each outcome, outcome followup time, and other useful information) were collected by 2 authors independently. After completing the forms, the disputes between them were resolved by discussion and by third party, if needed. In cases where the article information was incomplete and specific information about article was needed, the corresponding author was called and in the case of cooperation, necessary information was taken.

\section{Statistical Methods}

Since the data of studied outcomes were continuous, the mean difference or standardized mean difference and reverse variance method were used to pollute these data. The Fix model was the statistical model which was used in the first step. However, in cases where there was high heterogeneity between studies, a randomized model was used. The chi-square and $\mathrm{I}^{2}$ statistics were used for evaluating heterogeneity. The I 2 more than or equal to $40 \%$ indicated high heterogeneity and less than this value indicated negligible heterogeneity. ${ }^{12}$ In all tests, the significant level was considered to be 0.05 (95\% CI). The meta-analysis was performed using RevMan software, version 3.1.

\section{Results}

Study Selection and Characteristics of Included Studies As shown in Figure 1, in total, of 823 found papers, 171 duplicate articles were identified and deleted. Of 623 remaining articles, 519 papers were excluded based on studying titles and abstracts and 119 articles were excluded after studying the full text of articles. Finally, 14 clinical trial articles entered into the stage of quality

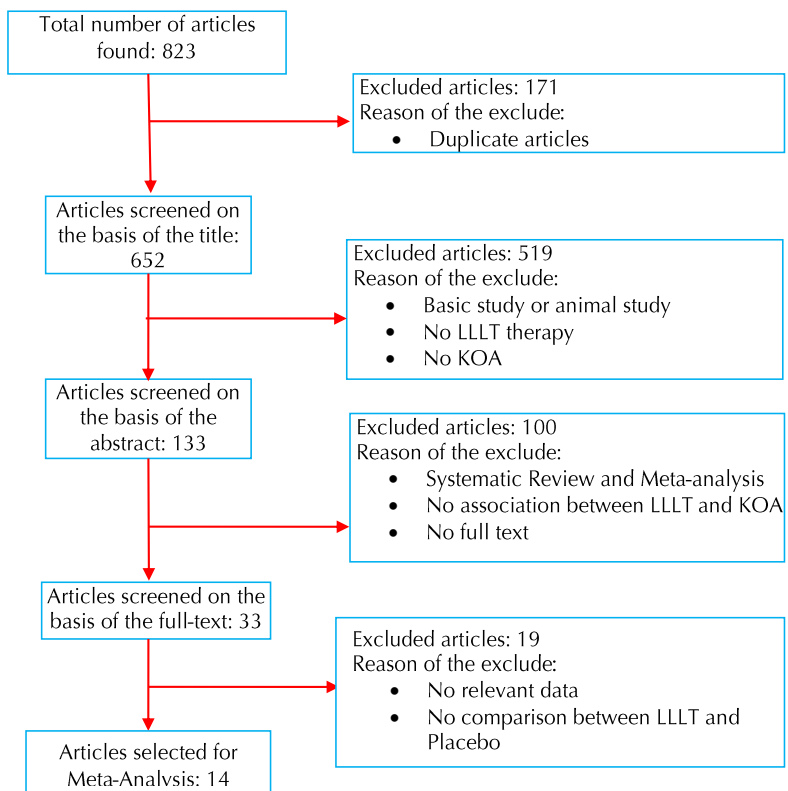

Figure 1. Search Results and Article Selection.

assessment and data extraction.

The total number of patient in LLLT group and placebo laser group were 350 and 328 respectively. The mean age of patients was between 40 to 90 years and follow-up time after treatment varied between 2 weeks to 1 year. All the including 14 studies were published between 1992 and 2016. The characteristics of the included studies are listed in Table 1.

\section{Study Quality}

Quality assessment of the articles showed that out of 14 articles, 9 had an acceptable quality, 2 had moderate quality and 3 had low quality (Figure 2).

\section{1-Pain Relief}

In this study, the result of pain relief was evaluated using VAS scale which is known as a tool for measuring the effectiveness of treatment. This scale is like a $10 \mathrm{~cm}$ ruler; one side of it indicates relief from pain and effectiveness of treatment and as we go to the other side of ruler, it indicates the presence of pain and at the end of which indicates that there is an intolerable pain. The scoring on this scale is done by patient him/herself. The patient him/ herself determines the amount of pain and scores it on ruler. ${ }^{27}$

\section{1-1-Pain at Rest}

Five studies ${ }^{14,16,17,19,24}$ including 579 patients (298 LLLT and 281 placebo) assessed the pain at rest outcomes of the patients. The overall inverse variance pooled SMD calculated for pain at rest was -0.45 (95\% CI; -0.84 , $-0.07)$ in favor of the LLLT ( $P=0.02$, Figure 3$)$. Test for heterogeneity was statistically significant $\left(I^{2}=80 \%\right.$, $P<0.00001)$. 
Table 1. Characteristics of the Articles Included

\begin{tabular}{|c|c|c|c|c|c|c|c|c|c|}
\hline \multirow{2}{*}{ Study } & \multirow{2}{*}{ Year } & \multirow{2}{*}{ Country } & \multicolumn{2}{|c|}{ Mean Age (SD) } & \multicolumn{3}{|c|}{ No. of Patients } & \multirow{2}{*}{ Follow up } & \multirow{2}{*}{ Reference } \\
\hline & & & LLLT & Placebo & LLLT & Placebo & Total & & \\
\hline Alfredo et al & 2012 & Brazil & $61.15(7.52)$ & $62.25(6.87)$ & 24 & 22 & 46 & $3 \& 11$ Weeks & 13 \\
\hline Alghadir et al & 2014 & Saudi Arabia & $55.2(8.14)$ & $57(7.77)$ & 20 & 20 & 40 & 4 Weeks & 14 \\
\hline Al Rashoud et al & 2014 & UK & $52(9)$ & $56(11)$ & 26 & 23 & 49 & $6 \& 24$ Weeks & 15 \\
\hline Fukuda et al & 2011 & Brazil & $63.0(9.0)$ & $63.0(8.0)$ & 25 & 22 & 47 & 3 Weeks & 16 \\
\hline Gur et al & 2003 & Turkey & $58.64(5.92)$ & $60.52(6.91)$ & 30 & 30 & 60 & $4,8, \& 12$ Weeks & 17 \\
\hline Hegedus et al & 2009 & Hungary & 49.48 & 49.48 & 18 & 9 & 27 & $2 \& 8$ Weeks & 18 \\
\hline Hinman et al & 2014 & Australia & $63.4(8.7)$ & $63.8(7.5)$ & 71 & 70 & 141 & 12 Weeks \& 1 year & 19 \\
\hline Kheshie et al & 2014 & Saudi Arabia & $56.56(7.86)$ & $55.6(11.02)$ & 20 & 20 & 40 & 6 Weeks & 20 \\
\hline Rayegani et al & 2012 & Iran & $61.7(2.9)$ & $61.2(7.2)$ & 12 & 13 & 25 & $4 \& 12$ Weeks & 21 \\
\hline Shen et al & 2009 & China & $60.1(6.83)$ & $56.4(7.41)$ & 20 & 20 & 40 & $2 \& 4$ Weeks & 22 \\
\hline Stelian et al & 1992 & Israel & $682(8.7)$ & $682(8.7)$ & 18 & 17 & 35 & 8 Weeks & 23 \\
\hline Tascioglu et al & 2004 & Turkey & $62.86(7.32)$ & $64.27(10.55)$ & 20 & 20 & 40 & $3 \& 24$ Weeks & 24 \\
\hline Youssef et al & 2016 & Saudi Arabia & $67.5(2.5)$ & $66.3(3.2)$ & 18 & 15 & 33 & 8 Weeks & 25 \\
\hline Yurtkuran & 2007 & Turkey & $51.83(6.83)$ & $53.478(7.13)$ & 28 & 27 & 55 & $2 \& 12$ Weeks & 26 \\
\hline
\end{tabular}

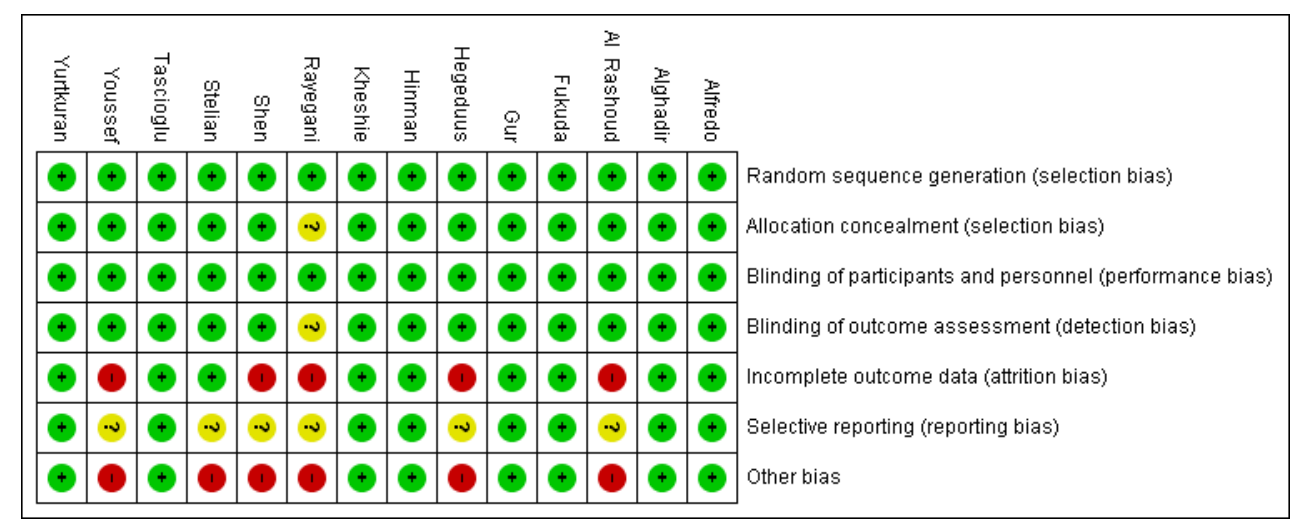

Figure 2. Risk of Bias Summary of Articles Included. (+ low risk bias, - high risk bias, ? unclear risk)

\section{1-2-Pain in Activity}

Six studies ${ }^{14,16,17,19,24,26}$ including 689 patients (354 LLLT and 335 placebo) assessed the pain in activity outcomes of the patients. The overall inverse variance pooled SMD calculated for pain in activity was -0.31 (95\% CI; -0.55, $-0.07)$ in favor of the LLLT $(P=0.01$, Figure 4$)$. Test for heterogeneity was statistically significant $\left(I^{2}=58 \%\right.$, $P=0.008)$.

\section{1-3-Pain Total}

Five studies ${ }^{13,15,19,20,23}$ including 478 patients (251 LLLT and 227 placebo) assessed the pain total outcomes of the patients. The overall inverse variance pooled SMD calculated for pain total was -0.41 (95\% CI; -0.77 , $-0.04)$ in favor of the LLLT $(P=0.03$, Figure 5$)$. Test for heterogeneity was statistically significant $\left(\mathrm{I}^{2}=72 \%\right.$, $P=0.0007)$.

\section{2- Knee Function}

In this study, the effectiveness of treatment in relation to knee function was evaluated based on WOMAC measurement tool. This questionnaire is known as a standard for assessing and monitoring KOA. It is based on Likert scale of 0 to $4(0=$ no pain, $1=$ mild pain, $2=$ moderate pain, $3=$ severe pain, and $4=$ intolerable or very severe pain) and is completed by patient him/herself. This questionnaire has 24 general questions which are divided into 3 subscales: 5 questions are related to pain, 2 questions are related to stiffness, and 17 questions which constitute main body of this questionnaire are related to function. The minimum score in this questionnaire is 0 and the maximum score is 96 . The maximum score in pain, stiffness, and function subscales is 20,8 , and 68 . In general, in this questionnaire, if the overall score or score in subscales will be closer to 0 , it will indicate the effectiveness of treatment. If the scores will be higher, it will indicate adverse knee conditions. ${ }^{28}$

\section{2-1-WOMAC Function}

Seven studies ${ }^{13,14,19,20,22,24,26}$ including 637 patients (334 LLLT and 303 placebo) assessed the WOMAC function outcomes of the patients. The overall inverse variance pooled SMD calculated for WOMAC function was -0.36 (95\% CI; $-0.64,-0.08)$ in favor of the LLLT $(P=0.01$, Figure 


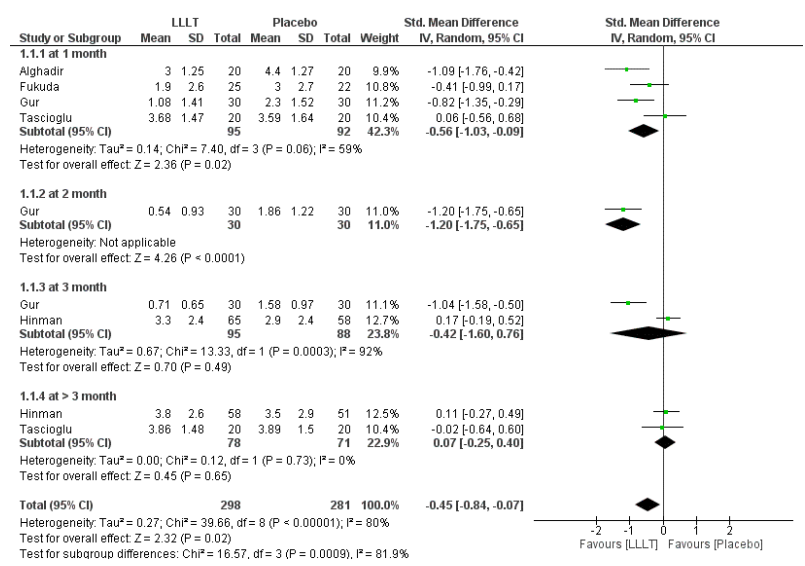

Figure 3. Forest Plot Analysis of the Pain at Rest.

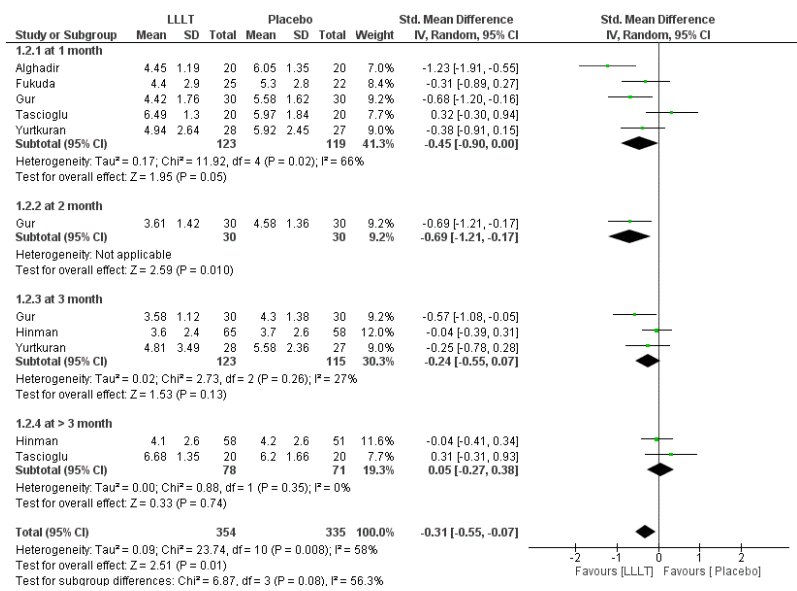

Figure 4. Forest Plot Analysis of the Pain in Activity.

6). Test for heterogeneity was statistically significant $\left(\mathrm{I}^{2}=\right.$ $65 \%, P=0.0009)$.

\section{2-2-WOMAC Pain}

Seven studies ${ }^{13,14,19,20,22,24,26}$ including 637 patients (334 LLLT and 303 placebo) assessed the WOMAC pain outcomes of the patients. The overall inverse variance pooled SMD calculated for WOMAC pain was $-0.2(95 \%$ CI; $-0.42,0.03)$. This difference was not statistically significant $(P=0.09$, Figure 7$)$. Test for heterogeneity was statistically significant $\left(\mathrm{I}^{2}=47 \%, P=0.04\right)$.

\section{2-3-WOMAC Stiffness}

Six studies ${ }^{13,27}$ including 405 patients (211 LLLT and 194 placebo) assessed the WOMAC stiffness outcomes of the patients. The overall inverse variance pooled SMD calculated for WOMAC stiffness was -0.23 (95\% CI; $-0.43,-0.03)$ in favor of the LLLT $(P=0.02$, Figure 8$)$. Test for heterogeneity was not statistically significant $\left(\mathrm{I}^{2}=0 \%\right.$, $P=0.65)$.

\section{2-4-WOMAC Total}

Seven studies ${ }^{13,14,17,19,22,24,26}$ including 585 patients (301 LLLT and 284 placebo) assessed the WOMAC total

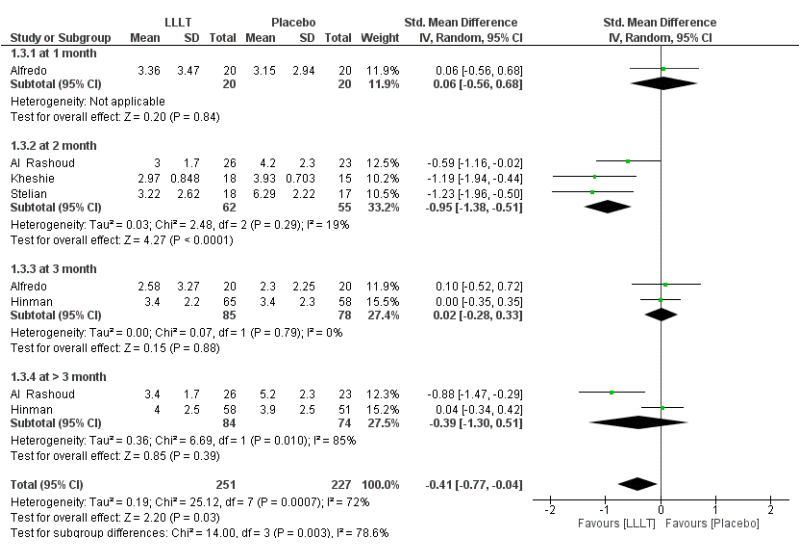

Figure 5. Forest Plot Analysis of the Pain Total.

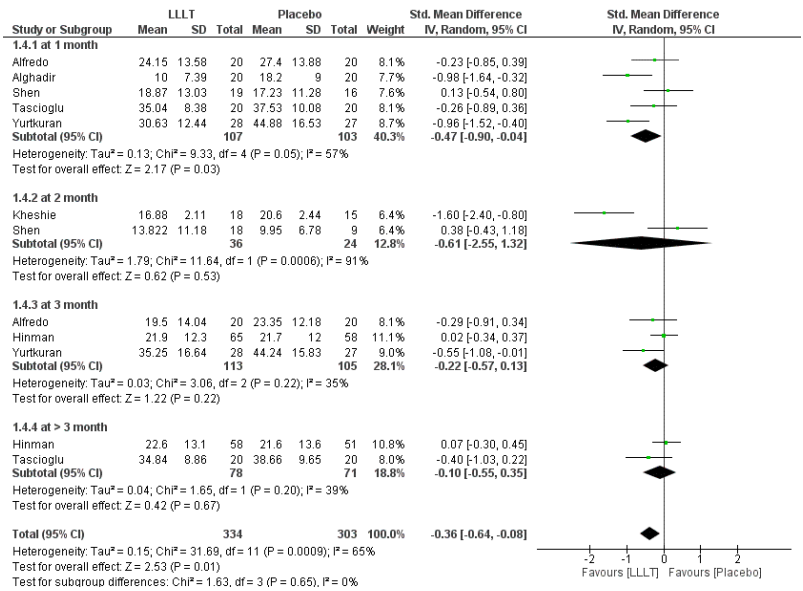

Figure 6. Forest Plot Analysis of the WOMAC Function.

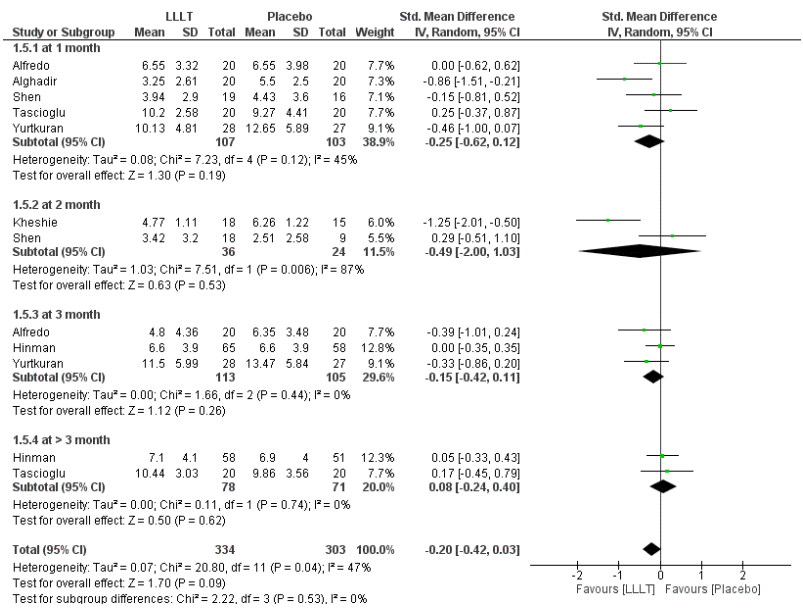

Figure 7. Forest Plot Analysis of the WOMAC Pain.

outcomes of the patients. The overall inverse variance pooled SMD calculated for WOMAC total was $-0.5(95 \%$ CI; - $0.73,-0.28)$ in favor of the LLLT $(P<0.0001$, Figure $9)$. Test for heterogeneity was statistically significant $\left(\mathrm{I}^{2}=\right.$ $45 \%, P=0.04)$. 


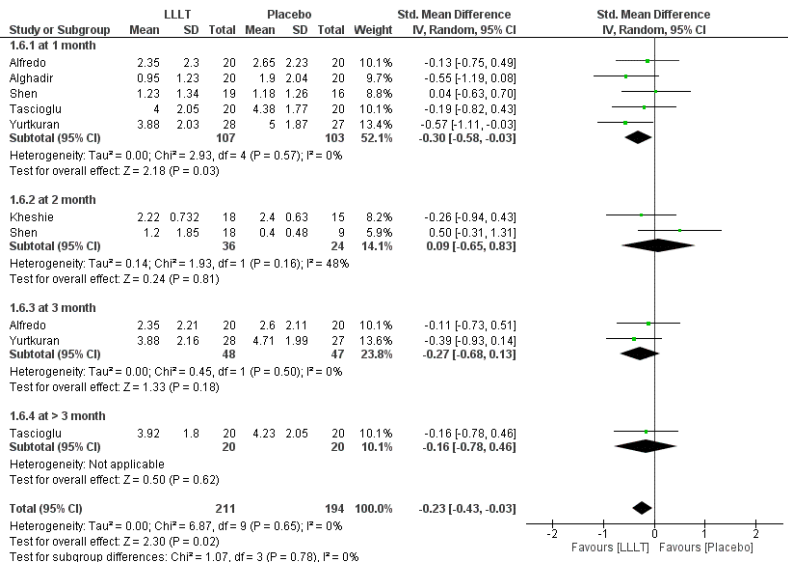

Figure 8. Forest Plot Analysis of the WOMAC Stiffness.

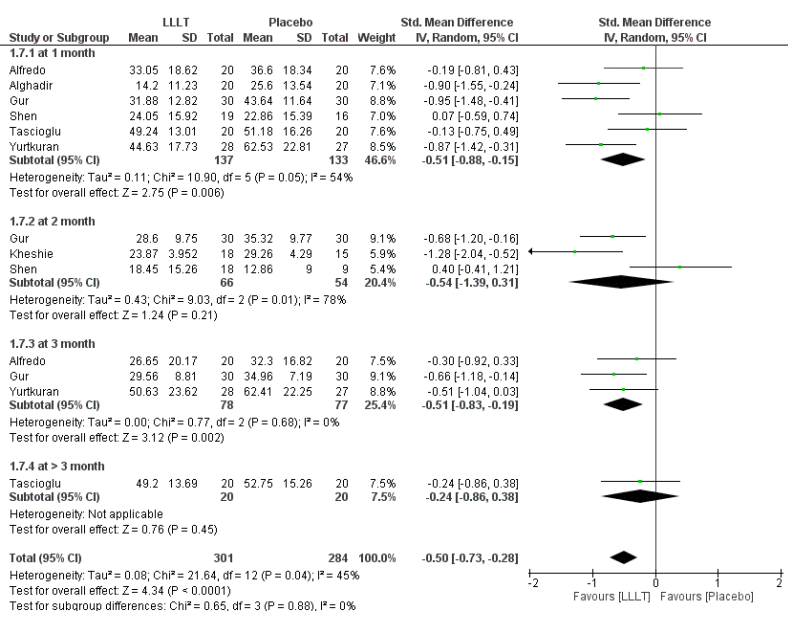

Figure 9. Forest Plot Analysis of the WOMAC Total.

\section{3- Range of Motion}

The recommended method of Marques was used to assess the effectiveness of knee range of motion (ROM). ${ }^{29}$

Four studies ${ }^{13,16,17,25}$ including 304 patients (137 LLLT and 167 placebo) assessed the range of motion outcomes of the patients. The overall inverse variance pooled SMD calculated for range of motion was 0.63 (95\% CI; - 0.13 , 1.4). This difference was not statistically significant ( $P$ $=0.1$, Figure 10). Test for heterogeneity was statistically significant $\left(\mathrm{I}^{2}=91 \%, P<0.00001\right)$.

\section{4- Side Effects}

Clinical trials which were analyzed in our study did not report any certain side effects of LLLT in patients.

\section{Discussion}

According to the World Health Organization's Functioning, Disability, and Health Classification model (ICF), the disability is the result of action-reaction between health conditions, environmental factors, and personal factors. According to this model, the disability results from damage in anatomical structure and physiological function of body, and limitations in activity and participation. The

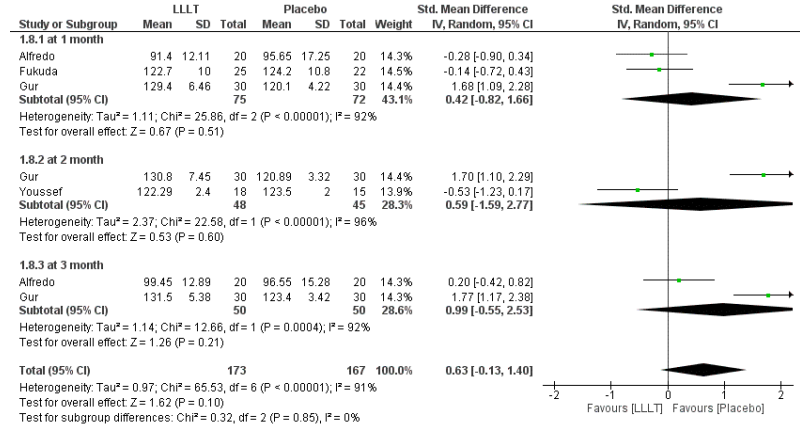

Figure 10. Forest Plot Analysis of the Range of Motion.

environmental and personal factors may play deterrent or facilitator role to affect the disabilities. KOA damages the structure and the physiological function of the knee joint. This damage may result in pain, functioning difficulties in daily living activities, and limited participation in living levels. On the other hand, environmental and personal barriers may increase the disability. LLLT has been introduced as a non-invasive therapeutic intervention for skeletal muscle disorders especially joint osteoarthritis during the past two decades. In spite of the widespread use of LLLT in KOA patients and also multiple trials in this area, there are inconsistent results regarding this technology efficacy. Stelian and colleagues ${ }^{23}$ research in 1992 was the first trial of laser effectiveness compared to placebo laser in KOA patients. The results of this trial showed improved function and pain reduction outcomes in patients who received laser treatment. After this trial, the studies conducted in the following years including Bulow et $\mathrm{a}^{30}$ and Tascioglu et $\mathrm{al}^{24}$ did not confirm the efficacy of LLLT compared to placebo laser. There were no significant differences between 2 groups in terms of performance improvement, pain relief and other clinical outcomes in these two studies. ${ }^{24,30}$ The efficacy of LLLT in KOA patients remains unknown due to these conflicting results. In spite of numerous clinical trials on LLLT efficacy in patients with KOA, there are few systematic and meta-analysis studies that clarify the LLLT efficacy and safety. This systematic and meta-analysis study was conducted based on the Cochrane Institute's guide. After reviewing titles, abstracts and full texts of 823 articles, 14 randomized clinical trials were randomly selected for analysis, of which, 9, 2 and 3 were of high, moderate, and low levels of quality, respectively. Total patients of LLLT group were 350 and total patients of placebo group were 328 . All outcomes that were analyzed were continuous. Therefore, data were pooled using random effect model and statistical inverse variance method and the standardized mean difference, which was statistically significant at the $5 \%$ level. The results of the metaanalysis on pain at rest, pain during activity, general pain, WOMAC function, WOMAC stiffness and overall WOMAC outcomes showed significant differences in favor of LLLT, but there were no statistically differences 
between the 2 groups in WOMAC knee pain and range of motion outcomes. There is insufficient evidence to draw any firm conclusions regarding the use of laser for the treatment of KOA. However, no side effects were reported and no evidence of harm was found.

Results of Huang and colleagues' ${ }^{10}$ meta-analysis did not show significant differences between two groups in overall pain outcomes, but our meta-analysis suggests a significant pain reduction in patients who received LLLT. Huang meta-analysis also showed no significant differences between two groups in WOMAC stiffness and WOMAC functional outcomes, while our metaanalysis indicated a significant improvement in patients who received LLLT. The results of our meta-analysis were similar to Huang and colleagues' study ${ }^{10}$ concerning WOMAC pain and range of knee motion outcomes in which there were no significant differences between two groups.

Since there was a significant heterogeneity in results of outcome meta-analysis, it cannot be said with certainty that the LLLT impacts in reducing pain and improving function in patients with KOA.

The use of different wavelengths, treatment times, number of sessions, and adjuvant therapy such as physical therapy along with LLLT, attrition during followup periods, illness severity, energy density and laser continuous output are the most important factors that affect the efficacy of Low Power Laser in patients with KOA. It seems that differences in these parameters are causing heterogeneity in the meta-analysis results. The wavelengths used in the entered trials was between 830 to $904 \mathrm{~nm}$ and the energy density was between 0.76 to $50 \mathrm{~J} / \mathrm{cm}^{2}$ as well as laser output varied between 4 to 800 $\mathrm{mW}$. It seems that the differences in wavelength, laser output and energy density in the entered trials may result in heterogeneity. The total numbers of treatment sessions in the underlying trials were between 8 to 20 sessions and the length of treatment times varied between 6 and 160 minutes and this probably caused the different results of the LLLT efficacy. On the other hand, if the illness severity is mild, treatment effectiveness will be more evident. So LLLT do not have enough effectiveness for severe cases. It seems that the application of LLLT combined with other methods such as physical exercises leads to better clinical outcomes. Most attrition during follow-up periods were related to studies of Shen et $\mathrm{al}^{22}$ ( 13 cases), Hinman et $\mathrm{al}^{19}$ ( 32 cases), Kheshie et $\mathrm{al}^{20}$ (7 cases) and Alfredo et $\mathrm{al}^{13}$ (6 cases). Reduced sample size may lead to biased results in these trials.

The last systematic study in this area in recent years belongs to Huang et al. ${ }^{10}$ However, the study neither encompasses some related articles, nor considers follow-up period of outcomes in the analysis, it also does not analyze consequences such as pain at rest, pain during activities and quality of life. The most important limitation of this study is, of course, high heterogeneity between studies that is the reason for using random model for meta-analysis. Moreover, another limitation of this study is small sample size in each paper and generally low sample size of outcomes which were analyzed. Three articles in reviewing of pain outcome, 2 articles in reviewing of activity outcome and 3 articles in reviewing of motion range reported just the mean without standard deviation and exact $P$ value. We tried to make contact with the corresponding authors of these trials. Unfortunately, we did not get any response. Incomplete statistical data caused data exclusion from analysis and this may affect the results.

\section{Conclusion}

The results of our systematic review and meta-analysis have provided the best current evidence on LLLT in the treatment of KOA. Although the heterogeneity of the results calls for caution in interpretation, LLLT seemed to be effective in reducing pain and improving function in patients with KOA. In spite of some positive findings, this meta-analysis lacked data on how LLLT effectiveness is affected with important factors: wavelength, energy density, continuous laser output, treatment duration, number of treatment sessions, lost to follow-up, severity of KOA and site of application.

\section{Ethical Considerations}

Not applicable.

\section{Conflict of Interests}

The authors have no conflict of interests relevant to this article to disclose.

\section{Acknowledgements}

The authors would like to thank the following individuals who have contributed at various stages through the development of this project.

\section{References}

1. Le TK, Montejano LB, Cao Z, Zhao Y, Ang D. Health care costs in US patients with and without a diagnosis of osteoarthritis. J Pain Res. 2012;5:23-30. doi:10.2147/JPR. S27275.

2. Badley EM, Rasooly I, Webster GK. Relative importance of musculoskeletal disorders as a cause of chronic health prob-lems, disability and health care utilization: findings from the 1990 Ontario Health Survey. J Rheumatol. 1994;21:505-514.

3. Chen D. Updated therapy in elderly patients with Knee osteoarthritis. Int J Gerontol. 2007;1(1):31-39. doi:10.1016/ s1873-9598(08)70021-8.

4. Gupta S, Hawker GA, Laporte A, Croxford R, Coyte PC. The economic burden of disabling hip and knee osteoarthritis (OA) from the perspective of individuals living with this condition. Rheumatology. 2005;44:1531-17. doi:10.1093/ rheumatology/kei049.

5. Bijlsma JW, Berenbaum F, Lafeber FP. Osteoarthritis: an update with relevance for clinical practice. Lancet. 2011;377(9783): 2115 -2126.

6. Melo MD, Pompeo KD, Brodt GA, Baroni BM, da 
Silva Junior DP, Vaz MA. Effects of neuromuscular electrical stimulation and low-level laser therapy on the muscle architecture and func-tional capacity in elderly patients with knee osteoarthritis: a randomized controlled trial. Clin Rehabil. 2015;29(6):570-580. doi: 10.1177/0269215514552082.

7. Yousefi-Nooraie R, Schonstein E, Heidari K, et al. Low level laser therapy for nonspecific low-back pain. Cochrane Database Syst Rev. 2008;16(2):Cd005107. doi: 10.1002/14651858.CD005107.pub4.

8. Farivar S, Malekshahabi T, Shiari R. Biological effects of low level laser therapy. J Lasers Med Sci. 2014;5(2):58-62 .

9. Herman JH, Khosla RC. In vitro effects of Nd:YAG laser radiation on cartilage metabolism. J Rheumatol. 1988;15(12):1818 - 1826.

10. Huang Z, Chen J, Ma J, Shen B, Pei F, Kraus V. Effectiveness of low-level laser therapy in patients with knee osteoarthritis: a systematic review and meta-analysis. Osteoarthritis Cartilage. 2015;23:1437-44. doi:10.1016/j.joca.2015.04.005.

11. Higgins JP, Green S. Cochrane Handbook for Systematic Reviews of Interventions 5.1.0. http://www.cochrane.org/ training/cochrane-handbook. Updated March 2011.

12. Higgins JP, Thompson SG, Deeks JJ, Altman DG. Measuring inconsistency in meta-analyses. BMJ. 2003;327(7414):557560.

13. Alfredo PP, Bjordal JM, Dreyer SH, et al. Efficacy of low level laser therapy associ-ated with exercises in knee osteoarthritis: a randomized double-blind study. Clin Rehabil. 2012;26(6):523-533.

14. Alghadir A, Omar MT, Al-Askar AB, Al-Muteri NK. Effect of low-level laser therapy in patients with chronic knee osteoar-thritis: a single-blinded randomized clinical study. Lasers Med Sci. 2014;29(2):749-755. doi:10.1007/s10103013-1393-3.

15. Al Rashoud AS, Abboud RJ, Wang W, Wigderowitz C. Efficacy of low-level laser therapy applied at acupuncture points in knee osteoarthritis: a randomised double-blind comparative trial. Physiotherapy. 2014;100(3):242-248.

16. Fukuda VO, Fukuda TY, Guimarães M, et al. Short-term effi cacy of low-level laser therapy in patients with knee osteoarthritis: a randomized placebo-controlled, doubleblind clinical trial. Rev Bras Ortop. 2015;46(5):526-533. doi: 10.1016/S2255-4971(15)30407-9.

17. Gur A, Cosut A, Sarac AJ, Cevik R, Nas K, Uyar A. Efficacy of different therapy regimes of low-power laser in painful osteoarthritis of the knee: a double-blind and randomized controlled trial. Lasers Surg Med. 2003;33(5):330-338.

18. Hegedus B, Viharos L, Gervain M, Galfi M. The effect of low-level laser in knee osteoarthritis: a double-blind, randomized, placebo-controlled trial. Photomed Laser Surg. 2009;27(4):577-584. doi:10.1089/pho.2008.2297.

19. Hinman RS, McCrory P, Pirotta M, et al. Acupuncture for chronic knee pain: a randomized clinical trial. JAMA. 2014;312(13):1313-1322.

20. Kheshie AR, Alayat MS, Ali MM. High-intensity versus low-level laser therapy in the treatment of patients with knee osteoarthritis: a randomized controlled trial. Lasers Med Sci. 2014;29(4):1371-1376. doi:10.1007/s10103-0141529-0.

21. Rayegani SM, Bahrami MH, Elyaspour D, Saeedi M, Sanjri H. Therapeutic effects of low level laser therapy (LLLT) in knee osteoarthritis, compared to therapeutic ultrasound. J Lasers Med Sci. 2012;3(2):71-74

22. Shen X, Zhao L, Ding G, et al. Effect of combined laser acupuncture on knee osteoarthritis: a pilot study. Lasers Med Sci. 2009;24:129-136.

23. Stelian J, Gil I, Habot B, et al. Improvement of pain and disability in elderly patients with degenerative osteoarthritis of the knee treated with narrow-band light therapy. $J$ Am Geriatr Soc. 1992;40:23-26.

24. Tascioglu F, Armagan O, Tabak Y, Corapci I, Oner C. Low power laser treatment in patients with knee osteoarthritis. Swiss Med Wkly. 2004;134(17-18):254- 8. doi:2004/17/ smw-10518.

25. Youssef EF, Muaidi QI, Shanb AA. Effect of Laser Therapy on Chronic Osteoarthritis of the Knee in Older Subjects. J Lasers Med Sci. 2016;7(2):112-129.

26. Yurtkuran M, Alp A, Konur S, Ozcakir S, Bingol U. Laser acupuncture in knee osteoarthritis: a double-blind, random-ized controlled study. Photomed Laser Surg. 2007;25(1):14-20.

27. Revill SI, Robinson JO, Rosen M, Hogg MI. The reliability of a linear analogue for evaluating pain. Anaesthesia. 1976; 31:1191-1198.

28. Bellamy N, Buchanan WW, Goldsmith $\mathrm{CH}$, Campbell J, Stitt LW. Validation study of WOMAC: a health status instrument for measuring clinically important patient relevant outcomes to antirheumatic drug therapy in patients with osteoarthritis of the hip or knee. J Rheumatol. 1988;15:1833-1840.

29. Marques AP. Manual de Goniometria. 2nd ed. Editora Manole; 2003.

30. Bulow PM, Jensen H, Danneskiold-Samsoe B. Low power $\mathrm{Ga}-\mathrm{Al}$-As laser treatment of painful osteoarthritis of the knee. A double-blind placebo-controlled study. Scand J Rehabil Med. 1994;-26(3):155-159. 